\title{
Quantitative study of bronchial mucous gland enlargement
}

\section{ANDREW N DOUGLAS}

\author{
From the Pathology Branch, Institute of Occupational Medicine, Edinburgh
}

ABSTRACT Histological sections from 30 lower lobe bronchi, taken from coalminers' lungs collec ted for the British National Coal Board's Pneumoconiosis Field Research, were selected according to the proportion of mucous gland area occupying the non-cartilaginous part of the bronchial wall. The total gland area expressed as a percentage of the non-cartilaginous wall was called the gland? index. Estimations were made of the total number of gland cells and acini on a section and of their $\vec{z}$ numbers per unit area of gland. These estimations were compared with the gland index. The tota numbers of gland cells and acini were found to be directly related to the gland index $(r=0.84$ and 0.86 ), whereas no relationship was found between the number of gland cells or acini per unit area of gland and the gland index $(r=0.08$ and 0.02$)$. This indicates that bronchial mucous glando enlargement is primarily a hyperplastic change. A direct relationship between the numbers of gland cells per unit area of gland and acini per unit area gland was found $(r=0 \cdot 69)$, and this suggests that acinar enlargement is likely to be caused by accumulation of mucus in the acinar lumen.

It has been recognised for many years that the enlargement of bronchial mucous glands is related to excessive production of mucus in chronic bronchitis. ${ }^{1-4}$ This enlargement has been loosely described as "hypertrophy" on many occasions but recently the term "hyperplasia" has been used. ${ }^{5}$

It is of interest to know whether the process of bronchial gland enlargement seen in chronic bronchitis is caused primarily by an increase in the size of the gland cells or by an increase in their number. These processes are called hypertrophy and hyperplasia respectively. Reid $^{1}$ concluded that hypertrophy was the dominant process but later results have not confirmed this. De Haller and Reid, ${ }^{6}$ for example, found no evidence that the mean acinar diameter increased in chronic bronchitis as might have been expected, and Bedrossian et $\mathrm{al}^{7}$ found little association between acinar density and gland size. This gap in the knowledge of the pathology of chronic bronchitis was commented on bv Thurlbeck. ${ }^{8}$

This paper contains the results of some work undertaken to clarify this aspect of bronchial gland enlargement.

Address for reprint requests: Mr AN Douglas, Pathology Branch, Institute of Occupational Medicine, 8 Roxburgh Place, Edinburgh EH8 9SU.

\section{Methods}

The bronchi examined formed part of a series obtained from the lungs of coalminers include of in the British National Coal Board's Pneumoconiosis Field Research. Three groups of $1 \overrightarrow{\mathrm{B}}$ lower lobe bronchi were selected according ton the proportion of mucous gland area occupying the non-cartilaginous part of the bronchial wall 3 . The ratio of gland area: non-cartilaginous walb area expressed as a percentage was called the gland index. The three groups of bronchi had gland indices of $\leqslant 15,16-25$, and $>25$.

The sections were cut at $3 \mu \mathrm{m}$ to minimis nuclear overlap, thereby facilitating the counting of nuclei. The sections were stained by Haes matoxylin with Van Gieson counterstain. Beforo examining the sections, their order waș randomised to minimise bias.

The following measurements were obtained from each section. (1) The area of the non? cartilaginous wall was measured from a drawing? of the projected image of the whole bronchus ${ }_{0}^{-}$ and will be called the "soft" wall in the text. (25 The total area of the bronchial glands wass measured from drawings made with the aid of a "camera lucida" microscope attachment. The drawings in both these two measurements were made on tracing paper and the relevant areas 
were measured using a point count grid placed under the paper. (3) The mean number of acini per unit area of gland was determined by the following method. A number of gland fields from each section were photographed and printed to give a magnification of $\times 240$. As many non-overlapping fields as possible were photographed, the number being governed by the area of gland on the section. A sampling square $(50 \times 50 \mathrm{~mm})$ drawn on a transparent sheet was placed over each photograph at two non-overlapping locations. To minimise selection bias the square was aligned to cover the same positions on each photograph. The number of acini falling within the square was counted and the average reading used to calculate the number of acini per $\mathrm{mm}^{2}$ of gland. Acini crossed by the north and west lines of the square were counted to lie in the square while those crossed by the south and east lines were not. (4) The mean number of mucous and serous gland cells per unit area of gland was determined by a similar method to that used for determining mean number of acini per unit area of gland except that a total photographic magnification of $\times 650$ was used. The same sampling grid $(50 \times 50 \mathrm{~mm})$ and sampling technique was used. Two bronchial sections were later omitted as the nuclei were indistinct because of autolysis.

Measurements (1) and (2) were combined as follows to give the gland index or proportion of wall occupied by glands:

$$
\text { Gland index }=\frac{\text { gland area } \times 100}{\begin{array}{c}
\text { non-cartilaginous } \\
\text { wall area }
\end{array}}
$$

An estimation of the number of cells in the complete section was calculated as follows: cell density (cells $\mathrm{mm}^{-2}$ gland) $\times$ total gland area $=$ total gland cells in bronchial section.

To allow for variations in bronchial dimensions between individuals the gland areas were adjusted to a "soft" wall area of $30 \mathrm{~mm} .{ }^{2}$ This figure was chosen as it approached the average value $\left(33.1 \mathrm{~mm}^{2}\right)$ for the cases in the study.

\section{Results}

A summary of the results is given in table 1. As the gland index increased, the "total cell number" increased but the acinar densities remained the same.

Figure 1 shows the linear relationship between the total gland cell number on the bronchial section and the gland index $(r=0.84)$. A
Table 1 Summary of results

\begin{tabular}{llll}
\hline & \multicolumn{3}{l}{ Gland index } \\
\cline { 2 - 4 } & $\leqslant 15$ & $15 \leqslant 25$ & $>25$ \\
\hline Area of gland on bronchial section (a)* & $3 \cdot 18$ & $5 \cdot 34$ & $9 \cdot 02$ \\
SD & 0.94 & $0 \cdot 40$ & $1 \cdot 15$ \\
Acinar density & 201 & 211 & 212 \\
SD & 51 & 34 & 37 \\
Number of gland cells in (a) & 4204 & 8690 & 14084 \\
SD & 1349 & 1836 & 3146 \\
Number of cases & 10 & 8 & 10 \\
\hline
\end{tabular}

*Corrected to soft wall area of $30 \mathrm{~mm}^{2}$.

Differences in the number of gland cells between gland index groups are statistically significant $(p<0.001)$.

similar relationship was found between "total acini" and gland index $(r=0.86)$.

Figure 2, however, fails to show any association between acinar density and the gland index, and this lack of association was also found between cell density and the gland index $(r=0.08$ and 0.02 respectively).

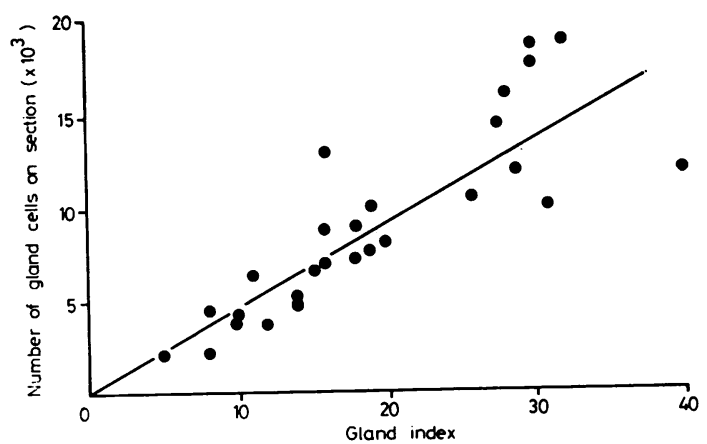

Fig 1 Total number of gland cells on a section plotted against the gland index $(r=0.84, p<0.001)$.

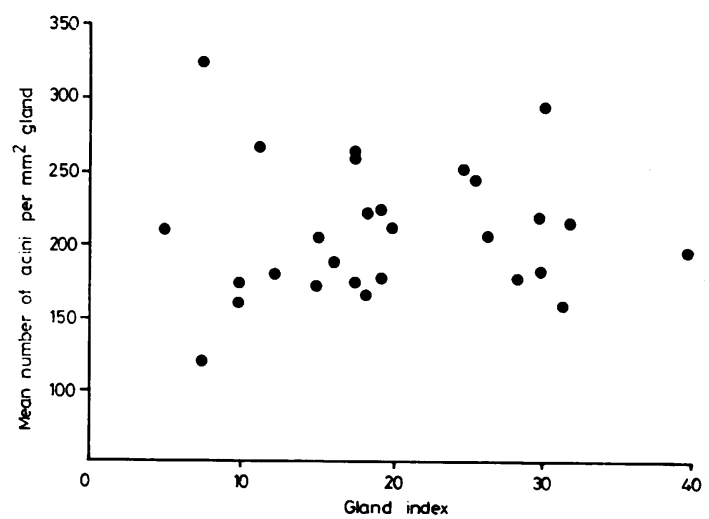

Fig 2 Mean number of acini per $\mathrm{mm}^{2}$ gland plotted against the gland index $(r=0.02, N S)$. 


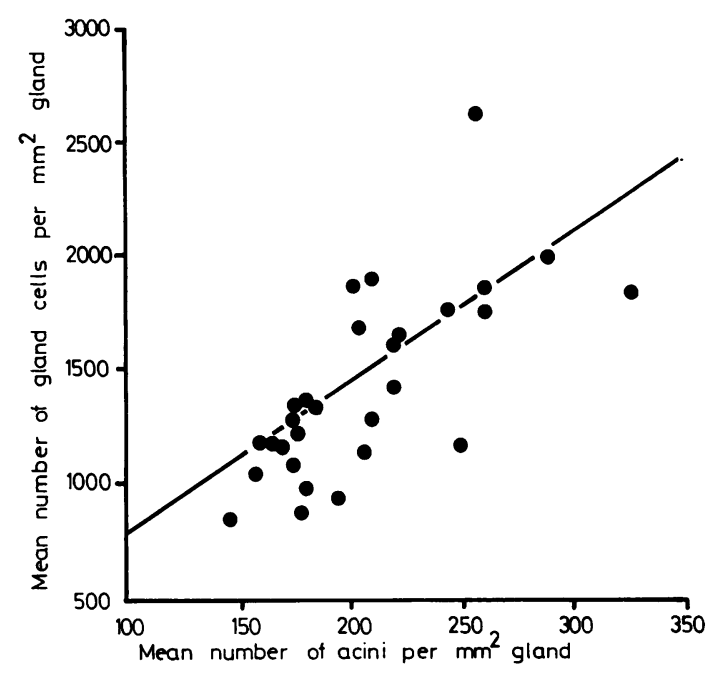

Fig 3 Relationship between the mean number of gland cells per $\mathrm{mm}^{2}$ gland and the mean number of acini per $\mathrm{mm}^{2}$ gland $(r=0.69, p<0.001)$.

Figure 3 shows that a decrease in acinar density was associated with a decrease in cell density $(r=0.69)$, suggesting hypertrophic changes. Hypertrophy, where present, however, was not associated with an increase in gland index (fig 2). Correlation coefficients were calculated for some of the pairs of variables and these are shown in table 2 .

\section{Discussion}

The results show that the dominant process in bronchial mucous gland enlargement is hyperplasia. The proportion of total gland area in the bronchial wall has been used as an index of gland enlargement previously, ${ }^{8-11}$ but in each case total bronchial wall was used. Restrepo and Heard ${ }^{4}$ found that the proportion of cartilage in the bronchial wall of large airways was unaffected in bronchitics, and so the use of the total wall area minus the area of cartilage has been used here in the calculation of the gland index to make it more sensitive to changes in the glands.

Table 2 Correlation coefficients between pairs of variables

\begin{tabular}{lllll}
\hline & Gland index & $p$ & Cell density & $p$ \\
\hline Cell number & 0.84 & $<0.001$ & - & - \\
Acinar number & 0.86 & $<0.001$ & - & - \\
Cell density & 0.08 & NS & - & - \\
Acinar density & 0.02 & NS & 0.69 & $<0.001$ \\
\hline
\end{tabular}

It is unfortunate that the clinical histories of the cases examined are not known in sufficien detail to classify them according to their bron흠 chitic symptoms. It has previously been shown $\frac{\bar{\sigma}}{\sigma}$ however, that the proportion of gland in theo bronchial wall increases in bronchitics, ${ }^{911}$ and $i{ }^{11}$ seems likely from the evidence presented herethat this increase is caused by a hyperplastic change of the glands. It is also possible that this type of change might be partly responsible for? the maintenance of bronchitic symptoms since $a_{2}^{2}$ hyperplastic change is less likely to revert to the original state than a hypertrophic change.

The results show that hypertrophic changes do $\overrightarrow{0}$ occur but are not obviously related to an increaseos in gland index. Although the number of cases examined was small, it appears that the relationship between cell density and acinar density is linear, the slope of the line representing the mean number of cells associated with an acinus? on the section. This suggests that acinar enlarge $\bar{\infty}$ ment may not be caused by an increase in gland cell size. If that was the case then it might be expected that the number of cell nuclei associated with an acinus would decrease as the acina density decreased. This is because compared tô the thickness of the section, an acinus is a large structure, and acinar density will be unaffected by section thickness. The density of nuclei, however을

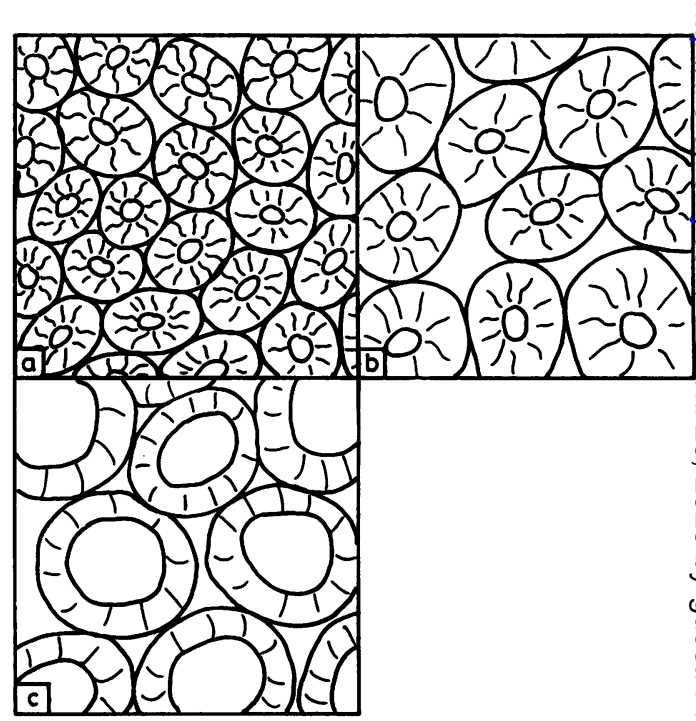

Fig 4 Schematic representation of two ways in which acinar enlargement may occur. (a) Normal acini in bronchial mucous gland; (b) acini enlarge by increasing cell size; (c) acini enlarged by increasing acinar lumen size. 
may be affected as the diameter of a nucleus is similar to the thickness of a section. If a hypertrophic change is present then as the volume of cell cytoplasm increases the probability of nuclei appearing in the section, and therefore the number of nuclei associated with any acinus, should decrease (fig 4). The data presented suggest that such reduction does not occur and thus that cellular hypertrophy is not the primary mechanism of acinar enlargement.

It remains a possibility that acinar enlargement may be caused by swelling of the acinar lumen as a result of mucus accumulation (fig 4). Where the glands are greatly enlarged the amount of mucus to be expelled through the gland duct is likely to be considerably increased and this may result in a back pressure which could swell the acinus. Alternatively a back pressure might result from alterations in the rheological properties of the mucus. Such changes in the properties of mucus have been reported ${ }^{12}$ but variation in the properties of mucus among bronchitics is considerable. ${ }^{13}$ Such changes in the properties of mucus have not been reported as being associated with gland size and could account for the variations in cell and acinar densities with gland index.

These results show that a major factor contributing to bronchial gland enlargement is an increase in cell number, and where acinar enlargement is seen this is likely to be caused by engorgement with mucus.

I would like to thank Dr David Lamb for his advice and $\mathrm{Mr} \mathrm{H}$ Tully, Mrs $\mathrm{K}$ Niven, and Mr D Brown for their skilled technical assistance. The financial support of the National Coal Board and the European Coal and Steel Community is acknowledged gratefully.

\section{References}

1 Reid L. Measurement of the bronchial mucous gland layer: a diagnostic yardstick in chronic bronchitis. Thorax 1960; 15:132-41.

2 Glyn AA, Michaels L. Bronchial biopsy in chronic bronchitis and asthma. Thorax 1960; 15: 142-53.

3 Thurlbeck WM, Angus GE, Paré JAP. Mucous gland hypertrophy in chronic bronchitis and its occurence in smokers. Br J Dis Chest 1963; 57: 73-8.

4 Restropo G, Heard BE. The size of bronchial glands in chronic bronchitis. J Pathol Bacteriol 1963; 85:305-10.

5 Macklem PT. The pathophysiology of chronic bronchitis and emphysema. Med Clin N Amer 1973; 57:669-79.

6 De Haller R, Reid L. Adult chronic bronchitis. Med Thorac 1965; 22:549-67.

7 Bedrossian CWM, Anderson AE, Foraker AG. Comparison of methods for quantitating bronchial morphology. Thorax 1971; 26:406-8.

8 Thurlbeck WM. Chronic airflow obstruction in lung disease. Philadelphia: WB Saunders, 1976: 44.

9 Dunnill MS, Massarella GR, Anderson AE. A comparison of the quantitative anatomy of the bronchi in normal subjects, in status asthmaticus, in chronic bronchitis and emphysema. Thorax 1969; 24:176-9.

10 Scott KWM. An autopsy study of bronchial mucous gland hypertrophy in Glasgow. Am Rev Respir Dis 1973; 107:239-45.

11 Takizawa T, Thurlbeck WM. A comparative study of four methods of assessing the morphological changes of chronic bronchitis. Am Rev Respir Dis 1971; 103:774-83.

12 Keal E, Reid L. Der Bronchialscheim-Seine Bildung, Zusammensetzung und Physikalischen Eigenschaften. Internist 1971; 12:416-24.

13 Dulfano MJ, Adler K, Philippoff W. Sputum viscoelasticity in chronic bronchitis. Am Rev Respir Dis 1971; 104:88-98. 